\title{
Searches for TeV Counterparts to Classical Gamma-Ray Bursts
}

\author{
V.Connaughton ${ }^{1}$, C.W.Akerlof ${ }^{3}$, S.Biller ${ }^{4}$, J.Buckley ${ }^{1}$, \\ D.A.Carter-Lewis ${ }^{5}$, M.Catanese ${ }^{5}$, M.F.Cawley ${ }^{6}$, D.J.Fegan ${ }^{2}$, \\ J.Finley $^{7}$, J.Gaidos ${ }^{7}$, A.M.Hillas ${ }^{4}$, R.C.Lamb ${ }^{5}$, R.Lessard ${ }^{2}$, \\ J.McEnery ${ }^{2}$, G.Mohanty ${ }^{5}$, N.A.Porter ${ }^{2}$, J.Quinn ${ }^{2}$, H.J.Rose ${ }^{4}$, \\ F.Samuelson ${ }^{5}$, M.S.Schubnell ${ }^{3}$, G.Sembroski ${ }^{7}$, R.Srinivasan ${ }^{7}$, \\ T.C.Weekes ${ }^{1}$, C.Wilson ${ }^{7}$, J.Zweerink ${ }^{5}$, S.Barthelmy ${ }^{8}$, T.Cline ${ }^{8}$. \\ N.Gehrels $^{8}$, G.J.Fishman ${ }^{9}$, C.Kouveliotou ${ }^{9}$, C.Meegan $^{9}$ \\ 1 Whipple Observatory, Harvard-Smithsonian CfA, U.S.A. \\ ${ }^{2}$ University College, Dublin, Ireland \\ 3 University of Michigan, U.S.A. \\ 4 University of Leeds, United Kingdom. \\ ${ }^{5}$ Iowa State University, U.S.A. \\ ${ }^{6}$ St.Patrick's College, Maynooth, Ireland \\ 7 Purdue University, U.S.A. \\ ${ }^{8}$ Goddard Space Flight Center \\ ${ }^{9}$ Marshall Space Flight Center
}

\begin{abstract}
Intense effort has gone into the observation of optical, radio and $\mathrm{X}$-ray GRB counterparts, either simultaneous to the burst or as quasisteady remnants. Here we report on a similar study at higher energies of $250 \mathrm{GeV}$ and above using ground-based telescopes. Imaging atmospheric Cherenkov telescopes have achieved great sensitivity and now complement observations by orbiting telescopes such as CGRO. Previous studies of bursts by the Whipple Collaboration (4) combined with recent improvements to the telescope, indicate that sensitivity to a fluence of $6 \times 10^{-9} \mathrm{erg}-\mathrm{cm}^{-2} \mathrm{can}$ be achieved. Observations by the Whipple Collaboration of nine BATSE positions, one within 2 minutes of the BATSE burst, using coordinates distributed through BACODINE will be reported. Analysis techniques will be described and an upper limit to the high-energy delayed or extended emission of observed candidates will be calculated.
\end{abstract}

\section{INTRODUCTION}

The recent technical advances represented by the atmospheric Cherenkov imaging technique (2) have opened up the field of gamma-ray astronomy above $250 \mathrm{GeV}$ and raised the possibility that these techniques can be used in exploring the gamma-ray burst phenomenon.

(C) 1996 American Institute of Physics 


\section{SCIENTIFIC JUSTIFICATION.}

The scientific benefit of the detection of a $\mathrm{TeV}$ component to a classical gamma-ray burst would be: a) it would really constrain the particle acceleration models for the sources and provide valuable insights into the source radiation mechanisms; b) it would set an upper limit to the distance to the source due to the predicted absorption of $\mathrm{TeV}$ photons by pair-production with the infra-red photons in intergalactic space (eliminate deep cosmological models) (7); c) it would give a good measure of the location of the burst; d) it would open a new window to the study of gamma-ray burst phenomena from the ground.

From the detection of some of the BATSE bursts by the EGRET detector at $\mathrm{GeV}$ energies an extrapolation to $\mathrm{TeV}$ energies may be made. A simple extrapolation from the observed delayed flux in the February 17, 1994 burst suggests a flux $>10^{4}$ photons. These bright bursts would be easily seen; fainter ones would also be detectable depending on their time structure and position location. The discovery of a delayed component in some bursts (6) by EGRET implies a long observation window may be available at high energies for at least some of the bursts observed by BATSE, so that response time does not need to be instantaneous.

\section{FOLLOW-UP OBSERVATIONS.}

Since May, 1994 we have been engaged in follow-up observations of BATSE bursts. The distribution of BATSE coordinates through BACODINE (1) make this project feasible. The Cherenkov telescope has maximum sensitivity at the zenith but can be used down to elevations of $20^{\circ}$ (as the elevation decreases the energy threshold increases as does the collection area); hence roughly $1 / 3$ of the sky is visible. With a BATSE detection rate of $1 /$ day, we can expect a burst within our accessible field of view at a rate of approximately $365 \times 0.12 \times 0.33 / 12$ or 1.2 per month. This rate is sufficiently high to justify observing and sufficiently low that it does not disrupt the routine observing program excessively.

The duty-cycle of the Cherenkov telescope is limited by sun, moon, cloud and instrument down-time to around $7 \%$. Some recent technical developments at the Whipple Observatory increase the feasibility of a burst detection. A filter has been found that permits the operation of the telescope under moonlight thus effectively doubling the duty-cycle (3). To reduce the response time between notice of a burst and the commencement of observations the azimuth drive of the telescope has been upgraded to increase the slew speed by a factor of five. With this improvement we are now able to slew the telescope to the burst position within five minutes of burst notification.

BACODINE notifications are processed as follows:

- BACODINE message is received by observer in dedicated email account. 
- Telescope slews to BACODINE coordinates for BATSE burst and tracks position for 28 minutes.

- Coordinates for 4 positions $3^{\circ}$ away from BACODINE position are calculated and each position is tracked for 28 minutes.

- Initial BACODINE position is tracked for 28 minutes.

- On another night, cycle is repeated for control purposes over the same range of telescope elevations.

- Following data reduction, events are selected on the basis of the shape of their image to reject hadronic events and other noise sources. Arrival directions of shape-selected events are determined.

- Excesses over the field-of-view of the control data are subtracted and plotted on a grid of $0.1^{\circ} \times 0.1^{\circ}$ bins.

Table 1 shows the Whipple BACODINE response; the analysis is presented in more detail in (5).

TABLE 1. Whipple Follow-ups to BACODINE Notifications

\begin{tabular}{||c|c|c|r|r|r|r|c||}
\hline \hline Date & $\begin{array}{c}\text { BATSE } \\
\text { Trig. }\end{array}$ & $\begin{array}{r}\text { BACODINE } \\
\text { Intensity } \\
\text { Cts/sec }\end{array}$ & $\begin{array}{r}\text { Distance } \\
\text { BACO- } \\
\text { Hunts. }\end{array}$ & $\begin{array}{r}\text { Dur. } \\
\text { BATSE } \\
\text { (sec) }\end{array}$ & $\begin{array}{r}\text { Whipple } \\
\text { delay } \\
\text { (min) }\end{array}$ & $\begin{array}{r}\text { Elev- } \\
\text { ation } \\
\text { (av) }\end{array}$ & $\begin{array}{c}\text { Cycles } \\
\text { observed } \\
(1-6)\end{array}$ \\
\hline 940516 & 2980 & 630 & N/A & N/A & 19 & $24-14$ & 1 (1 hour) \\
950208 & 3408 & 778 & 1.64 & N/A & 16 & N/A & 1,2 \\
950405 & 3494 & 704 & 9.5 & N/A & 8 & $27-33$ & $1,2,3+$ cont \\
950524 & 3598 & 8726 & 1.37 & 6 & 5 & $56-31$ & complete \\
950625 & 3649 & 1661 & 3.84 & 40 & 18 & $28-41$ & $1-6$ \\
950701 & 3658 & 9134 & 2.3 & 15 & 56 & $41-69$ & complete \\
951117 & 3909 & 1955 & 10.1 & 25 & 5 & $30-24$ & 1 (1 hour) \\
951119 & 3911 & 801 & 6.98 & 60 & 20 & $45-54$ & complete \\
951124 & 3918 & 1231 & 5.98 & 150 & 2 & $59-76$ & complete \\
\hline
\end{tabular}

\section{RESULTS}

To assess the sensitivity of the technique, observations of the Crab with the source at a position $0.4^{\circ}$ offset from center of camera were analyzed using the method described above. A $5 \sigma$ excess is seen from one 28 minute ON-OFF pair in the offset position as shown in Figure 1. Similar plots were produced for each of the 6 ON/OFF pairs acquired during BACODINE follow-ups, and no excesses above $3 \sigma$ were seen in any positions. Two of the contour plots are shown in Figure 2. Assuming a Crab-like spectrum for any BACODINE burst emission above $0.3 \mathrm{TeV}$, and taking the Crab fluence over 28-minutes as an upper limit to the excess from any BACODINE position, one can calculate 


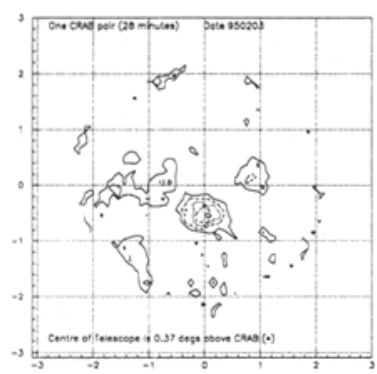

FIG. 1. One 28 minute ON/OFF pair on offset Crab shows sensitivity of technique.
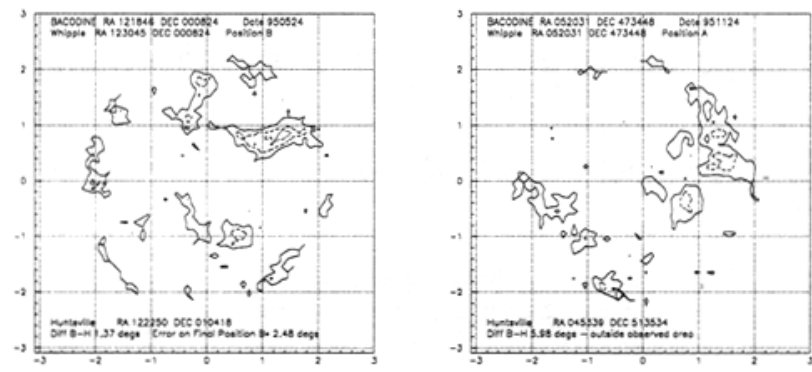

FIG. 2. Examples of 28 minute scans following burst 3598 and 3649 .

an upper limit to delayed or extended $\mathrm{TeV}$ flux over a 28-minute scan of $1.13 \pm 0.06 \times 10^{-7} \mathrm{erg} \mathrm{cm}^{-2}$ for a burster $0.4^{\circ}$ from the center of the field of

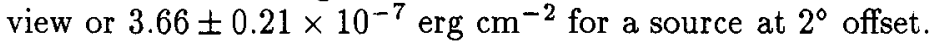

Shape-selected event rates show no variations of emission over a time-scale of minutes in any of the 28 minute scans.

Acknowledgements: We thank Scott Barthelmy and the BACODINE team for their support. This work is supported by US Department of Energy and NASA.

\section{REFERENCES}

1. S. Barthelmy, et al., in Gamma-Ray Bursts, eds. G.J. Fishman, J.J. Brainerd \& K. Hurley, AIP Conf.Proc. 307643 (AIP, New York, 1994).

2. M.F. Cawley, and T.C. Weekes, Exp. Ast. 6, 7 (1995).

3. M. Chantell, et al., Proc. 23rd ICRC 2, 544 (1995).

4. V. Connaughton, et al., in Gamma-Ray Bursts, eds. G.J. Fishman, J.J. Brainerd \& K. Hurley, AIP Conf.Proc. 307470 (AIP, New York, 1994).

5. V. Connaughton, et al., in preparation (1996).

6. K. Hurley, et al., Nature 372, 652 (1994).

7. F.W. Stecker, et al., ApJ 390, L49 (1992). 